\title{
WR-2721 (Amifostine) Ameliorates Cisplatin-Induced Hearing Loss But Causes Neurotoxicity In Hamsters: Dose-Dependent Effects
}

\author{
Michael W. Church, ${ }^{1,2,3}$ Brian W. Blakley, ${ }^{3,4}$ Don L. Burgio, ${ }^{2}$ and Anil K. Gupta ${ }^{2}$ \\ ${ }^{1}$ Department of Obstetrics and Gynecology, Wayne State University School of Medicine, Detroit, MI 48201, USA \\ ${ }^{2}$ Department of Otolaryngology and Head/Neck Surgery, Wayne State University School of Medicine, Detroit, MI 48201, USA \\ ${ }^{3}$ Karmanos Cancer Institute, Wayne State University School of Medicine, Detroit, MI 48201, USA \\ ${ }^{4}$ Department of Otolaryngology, University of Manitoba Winnepeg, Winnepeg, Manitoba, Canada R3A 1R9
}

Received: 1 April 2003; Accepted: 3 February 2004; Online publication: 30 July 2004

\section{ABSTRACT}

Chemoprotective agents reduce the toxic side effects of chemotherapy agents such as cisplatin. The conventional belief is that the chemoprotective agent WR-2721 (Amifostine), while protecting against most cisplatin-induced side effects, does not protect against cisplatin-induced ototoxicity (i.e., hearing loss). There is no knowledge, however, about the efficacy of high doses of WR-2721 (WR) in possibly protecting against cisplatin-induced ototoxicity. Thus, the dose-dependent effects of WR in possibly ameliorating cisplatin-induced ototoxicity were investigated. Hamsters were given a series of 5 cisplatin injections $(3 \mathrm{mg} / \mathrm{kg} /$ injection once every other day, i.p.) either alone or in combination with $18,40,80$, or $400 \mathrm{mg} / \mathrm{kg} /$ injection of the rescue agent WR $(n=5$ or $10 /$ group). Other groups received either $80 \mathrm{mg} / \mathrm{kg} /$ injection WR alone $(n=5)$ or were untreated $(n=14)$. Ototoxicity was assessed by auditory brain stem responses (ABR). WR provided dose-dependent rescue from cisplatin's ototoxicity with no protection at the low dose of $18 \mathrm{mg} /$ $\mathrm{kg}$, moderate protection at $40 \mathrm{mg} / \mathrm{kg}$, and nearly complete protection at 80 and $400 \mathrm{mg} / \mathrm{kg}$. However, WR doses of $40 \mathrm{mg} / \mathrm{kg}$ or higher caused neurotoxicity as evidenced by prolongations in the ABR's interpeak latencies. Thus, high doses of WR provided the beneficial effect of protecting against cisplatin-

Correspondence to: Michael W. Church - C.S Mott Center - 275 E. Hancock Ave. - Detroit, ML 48201. Telephone: (313) 577-1184; fax: (313) 577-8554; email: mchurch@med.wayne.edu induced ototoxicity, but had the harmful side effect of neurotoxicity. Previous failures to find chemoprotection from cisplatin-induced ototoxicity were likely due to the use of WR doses that were too small. The clinical implications of the beneficial and harmful effects of high doses of WR are discussed.

Keywords: amifostine, auditory brain stem response (ABR), cancer therapy, chemoprotection, cisplatin, neurotoxicity, ototoxicity, sensorineural hearing loss, WR-2721

\section{INTRODUCTION}

Cisplatin (CIS), or cis-diamminedichloroplatinum, is a chemotherapy agent widely used to treat cancers of the head, neck, ovaries, uterus, cervix, breast, testes, prostate, stomach, bladder, lungs, and brain (Loehrer and Einhorn 1984). One mechanism of cisplatin's antitumor action involves its binding to nuclear DNA molecules at sulfhydryl sites, resulting in a cascade of events that culminate in cell death. Cisplatin also binds to specific sites on the cell membrane, cytoplasm, and other non-DNA targets (Fuertes et al. 2003). As reviewed by Campbell et al. (2003a), another mechanism of cisplatin's toxicity involves the induction of reactive oxygen species (ROS) and the possible depletion of antioxidants such as glutathione.

Unfortunately, CIS's therapeutic potential is limited by its ototoxicity, neurotoxicity, nephrotoxicity, 
and hematotoxicity (Gandara et al. 1990). These toxic side effects can preclude the delivery of an adequate total dose and thereby limit CIS's therapeutic potential. The continuing use of CIS as a chemotherapy agent necessitates research on ways to ameliorate these side effects through the use of chemoprotective ("rescue") agents.

CIS distributes itself to nearly all tissues (e.g., Litterst et al. 1979; Poirier et al. 1992). In the nervous system, platinum concentrations were found to be relatively low in the brain, but relatively high in the sural nerves and spinal ganglia. This may explain why peripheral neuropathy is a major side effect of CIS treatment (Thompson et al. 1984). In the auditory system, the distribution and toxic effects of platinum in the auditory nerve and auditory brain stem nuclei have not been investigated. It is well known, however, that the inner ear is a major target of cisplatin. Platinum concentrations were found to be relatively high in the vestibular labyrinthine tissue, basilar membrane, and stria vascularis in particular (Schweitzer et al. 1986). The main structural effects of CIS's toxicity in the inner ear include damage to the stria vascularis, the organ of Corti, Reissner's membrane, and the outer hair cells. The behavioral effects of CIS's ototoxicity include sensorineural hearing loss and tinnitus (see reviews by Campbell et al. 2003a; Schweitzer 1993). These toxic effects adversely affect the patient's quality of life. Thus, ameliorating CIS's ototoxicity is an important health-related goal.

Several thiol-containing drugs have demonstrated rescue properties, particularly in terms of reducing CIS's nephrotoxicity. Such drugs include sodium thiosulfate (STS) (Pfeifle et al. 1985), diethyldithiocarbamate (DDTC) and WR-2721 (WR) (Gandara et al. 1990). The present study is focused on WR. WR is an organic thiophosphate whose metabolite WR-1065 is more readily absorbed by normal versus malignant cells. Protection from CISinduced damage then occurs from this metabolite's various antioxidant actions against ROS and through proton donation to damaged DNA at the sulfhydryl sites (Culy and Spencer 2001; Treskes et al. 1993). WR has been effective in ameliorating CIS-induced hematotoxicity and nephrotoxicity (Glover et al. 1986) and neuropathy (Mollman et al. 1988). WR's success in reducing CIS-induced ototoxicity during clinical trials is mixed, however. The conventional belief is that WR offers little or no protection against CIS-induced ototoxicity. For example, one study reported results suggesting some mild amelioration of ototoxicity (Rubin et al. 1995) whereas most other studies did not (Gandara et al. 1991; Glover et al. 1987; Kemp et al. 1990; Petrilli et al. 2002; Rick et al. 2001). This area of literature has several shortcomings however. For example, most of the clinical studies failed to use appropriate comparison groups. Moreover, human and animal studies have failed to explore the possibility that high doses of WR might provide some degree of rescue from CIS-induced ototoxicity. Thus, there is a serious lack of knowledge about whether WR can reduce CIS-induced ototoxicity.

In two previous studies using hamsters, we found that STS and DDTC ameliorated CIS's ototoxicity and mortality, but WR did not (Church et al. 1995; Kaltenbach et al. 1997). Our WR dose selection (18 mg/ $\mathrm{kg}$ ) was extrapolated from human studies (Glover et al. 1986, 1987). It is possible that our failure to ameliorate CIS's ototoxicity was due to using a dose that was too small for the hamster. This possibility is underscored by recent mouse studies on chemoprotection from nephrotoxicity, hematotoxicity, gastrointestinal toxicity, and mortality. These studies used WR doses of 200-525 mg/ $\mathrm{kg}$ (Ito et al. 1994; Peters et al. 1992; van der Wilt et al. 1992). Consequently, the present study investigated whether high WR doses would provide amelioration of CIS's ototoxicity and mortality. Moreover, there is no knowledge about whether WR causes neurotoxicity. Thus, another goal was to assess WR's possible neurotoxic side effects.

Ototoxicity and neurotoxicity in the present study were assessed by the auditory brain stem response (ABR). The ABR was chosen as the dependent measure because of its proven sensitivity in assessing CISinduced hearing loss and the efficacy of chemoprotective drugs (e.g., Campbell et al. 2003a, 2003b; Church et al. 1995; Schweitzer 1993; Schweitzer et al. 1986). The hamster was chosen as the animal model because it has proven to be an excellent model of CIS-induced hearing loss. Moreover, the present study was designed to be a followup and extension of our previous studies that used hamsters (Church et al. 1995; Kaltenbach et al. 1997).

\section{MATERIALS AND METHODS}

\section{Subjects and treatment}

Wayne State University's animal investigation committee approved the procedures for this study. Institutional and NIH guidelines were followed.

This study used 54 male Syrian golden hamsters (Charles River Laboratories), aged 45-55 days at the start of treatment. Only males were used to avoid possible changes in the ABR (Elkind-Hirsch et al. 1994) and drug metabolism (Anthony and Berg 2002) associated with the female hormonal cycle. The male hamsters were randomly assigned to one of six drug treatment conditions or an untreated control group. Some hamsters were given a series of 5 CIS injections $(3 \mathrm{mg} / \mathrm{kg} /$ injection once every other day, 
i.p.) either alone $(n=10)$ or in combination with 18 , 40,80 , or $400 \mathrm{mg} / \mathrm{kg} /$ injection (i.p.) of WR that preceded the CIS injections by $30 \mathrm{~min}(n=10,5,5$, and 5 , respectively). The effects of WR alone on the ABR have never been investigated. Thus, another treatment group received the series of $80 \mathrm{mg} / \mathrm{kg}$ / injection of WR alone and served as a comparison group to assess any WR-induced toxicity $(n=5)$. An untreated control group, to which all other groups were compared, was also assessed $(n=14)$. Thirty days after the last injection, at ages $80-90$ days, the treated animals were assessed for ototoxicity using ABRs. These treatment regimens were based on our prior research experience (Church et al. 1995). The treatment groups will hereafter be referred to as CISalone, CIS + WR18, CIS + WR40, CIS + WR80, CIS + WR400, WR80-alone, and Control.

The animals in the Control, CIS-alone, and CIS + WR18 groups were a part of our previous studies (Church et al. 1995; Kaltenbach et al. 1997). Reusing their data for the present study was consistent with the principle of "reduction in the number of animals used" as advocated by Russell and Burch (1959). A period of 22 months separated the testing of the two animal colonies. All animals were bought from the same vendor (Harlan-Sprague-Dawley) and were matched for age and weight range. Both animal colonies were treated and tested in the exact same manner and conditions.

\section{ABR procedure}

Prior to ABR recording, each animal was given 100$150 \mathrm{mg} / \mathrm{kg}$ of the anesthetic ketamine (i.p.) plus 5$10 \mathrm{mg} / \mathrm{kg}$ xylazine (i.p.). Ketamine can influence the rodent ABR latencies and/or amplitudes, but the effects are minor and more importantly the thresholds are not altered and ABR quality is excellent (Church and Gritzke 1987). Rectal temperature was monitored because a decrease of $0.5^{\circ} \mathrm{C}$ can alter the ABR (Rossi and Britt 1984). A water-circulating heating pad maintained normothermia.

The ABR was differentially recorded between two subcutaneous platinum E-2 needle electrodes. The active (noninverting) electrode was inserted at the vertex, the reference (inverting) electrode behind the left ear, and the ground electrode behind the right ear. Evoked potentials were collected by a Bio-logic Navigator (Bio-logic Systems Corp, Mundelein, IL) and amplified by a factor of 300,000 times with a digital bandpass of 300-3000 Hz. At least 256 responses were averaged. The amplified signals were averaged with positivity displayed upward and traces stored on computer disk for later analysis. ABR activity was sampled at a rate of $0.02 \mathrm{~ms}$ per address. The analysis epoch was $10.24 \mathrm{~ms}$. An artifact rejection system eliminated individual responses if they contained voltages exceeding $\pm 8.2 \mu \mathrm{V}$.

Recordings were made in an electrically shielded, double-walled sound attenuation chamber (Allotech, Inc., Raleigh, NC). Binaural "open field" tone pips of $20,000,16,000,8000,4000$, and $2000 \mathrm{~Hz}$ were created by a stimulus generator (Modular Instruments, Inc., Malvern, PA) and delivered through a TDH-39P headphone positioned directly in front of the animal (tone burst rise $/$ fall time $=0.5 \mathrm{~ms}$, plateau $=10.0 \mathrm{~ms}, \quad$ polarity $=$ alternating,$\quad$ repetition rate $=12.5 / \mathrm{s}$ ).

ABR thresholds and latency-intensity profiles were determined by the method of limits (Church and Shucard 1986). Here, serial ABRs were gathered to a range of stimulus intensities starting at $100 \mathrm{~dB}$ peakequivalent sound pressure level (peSPL), then descending to $80,60,50,40,35,30,25,20$, and $15 \mathrm{~dB}$ peSPL as the ABR threshold was reached and passed. To establish ABR threshold more precisely, 2 and $3 \mathrm{~dB}$ changes in stimulus intensity levels were tested around the ABR's threshold (as determined by visual detection) and multiple ABR traces (2-4) were collected at each near-threshold intensity level. Then, an ascending series of ABRs was gathered starting below this point and moving up in stimulus intensity. Threshold was defined as the lowest intensity to elicit a reliably scorable ABR component. Stimulus intensity and frequency were measured at the animal's pinnae with a Bruel \& Kjaer sound level meter and an oscilloscope.

The hamster ABR is composed of four or five vertexpositive components (labeled P1 to P5) occurring within $6 \mathrm{~ms}$ of stimulus onset (Church et al. 1995). Although the neurogenerators of the hamster's ABRs have not been determined, in the mouse they probably reflect neural activity chiefly from the auditory nerve (P1), the posterior anteroventral cochlear nucleus (P2), the anterior anteroventral cochlear nucleus and the trapezoid body (P3), the medial superior olivary nucleus (P4), and the lateral lemniscus and/or inferior colliculus (P5) (Parham et al. 2001). The ABR's neurogenerators of higher-order mammals are more complex (Moller and Jeanetta 1985). The latency of each ABR component was measured as the time from the computer's triggering of the earphone to a wave's positive peak, including a $0.3 \mathrm{~ms}$ acoustic transit time between the earphone and the animal's pinnae. An experimenter, who was "blind" as to each animal's treatment condition, scored the ABR latencies and thresholds. A second experimenter then checked the ABR scoring for reliability purposes.

\section{Data analysis}

Analyses of variances (ANOVA) were used to assess the statistical significance. If an ANOVA indicated 
A. Control

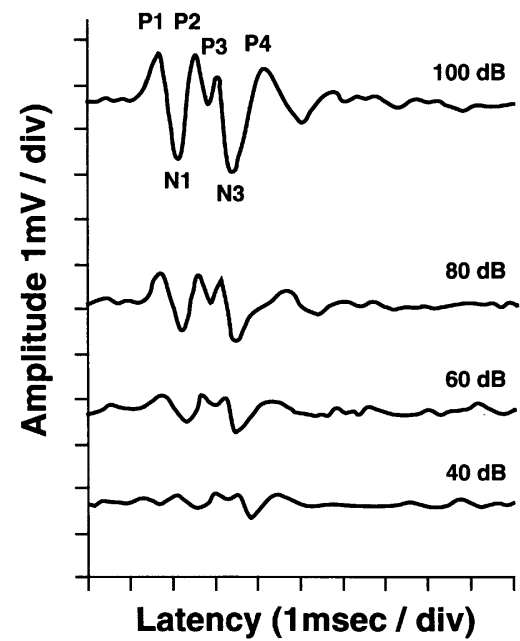

B.

CIS-Alone

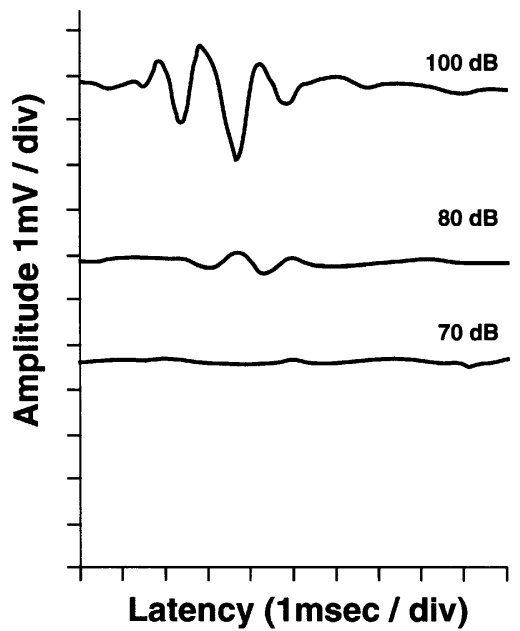

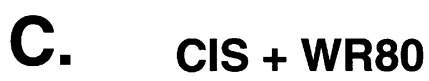

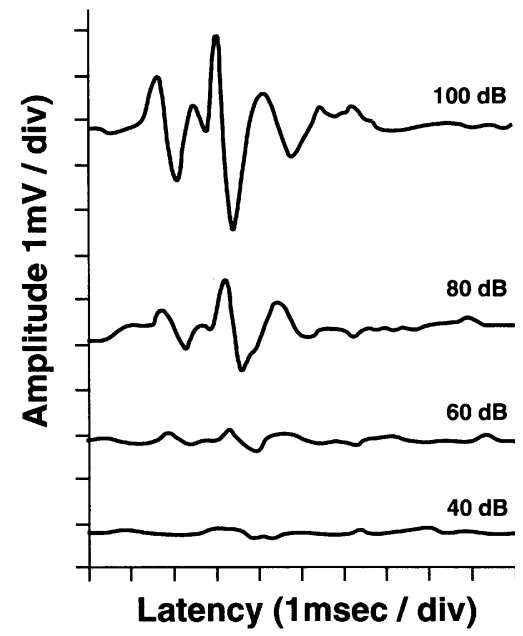

FIG. 1. Serial $A B R s$ evoked by $16,000 \mathrm{~Hz}$ tone pips. A. ABRs from a typical Control animal with an ABR clearly visible at $40 \mathrm{~dB}$. B. ABRs from a typical CIS-treated animal with reduced amplitudes, prolonged latencies, and threshold elevated to about $80 \mathrm{~dB}$. C. ABRs

significant group difference, a post hoc test (Duncan's Multiple Range test) was used to make pairwise comparisons between treatment groups. The criterion for statistical significance was $p \leq 0.05$.

\section{RESULTS}

\section{Mortality}

None $(0 \%)$ of the animals in the Control, WR80alone, CIS + WR400, or CIS + WR80 groups died during treatment. Twenty percent $(20 \%)$ of the animals in each of the CIS + WR40, CIS + WR18, and CIS-alone groups died during treatment from CISrelated toxicity as evidenced by severe lethargy and weight loss before death. One animal in the CIS + WR400 group died during ABR testing, ostensibly from the anesthetic injection. This animal's death was therefore not considered as a CIS-related or WR-related mortality. We cannot rule out the possibility that the CIS or WR treatments weakened this animal's health to the point that it was more susceptible to the anesthetic's lethality. Statistical analysis (Fisher's exact test) indicated a trend for a reduction in mortality at WR doses of $80 \mathrm{mg} / \mathrm{kg}$ or greater $(p=0.098$; 2-sided probability level).

\section{ABR morphology, serial ABRs}

Figure 1 provides examples of serial ABRs to the $16,000 \mathrm{~Hz}$ tone pips collected from typical animals in from a typical CIS + WR80 animal with preservation of amplitudes and threshold. The ABRs from the Control animal were adapted from a previous publication (Church et al. 1995).

the Control, CIS, and CIS + WR80 groups. These ABRs comprised four vertex-positive waves, labeled P1 through P4. The most prominent vertex-negative waves, N1 and N3, are also labeled in Figure 1. ABR wave amplitudes decreased and their latencies prolonged as stimulus intensity decreased, in accordance with prior studies (Church et al. 1995; Church and Shucard 1986). The ABR threshold was the lowest stimulus intensity to produce a scorable ABR wave. As seen in Figure 1, the CIS animal had an elevated ABR threshold compared with the Control animal. The CIS + WR80 animal, on the other hand, had a nearnormal ABR threshold.

\section{ABR thresholds}

ABR thresholds provide measures of auditory sensitivity. If a control group has an average ABR threshold of $30 \mathrm{~dB}$ to a tone pip of a given frequency and if a treated animal has a threshold of 60 $\mathrm{dB}$, then the treated animal has a threshold elevation of $30 \mathrm{~dB}$. This threshold elevation approximates the degree of hearing loss (Jacobson 1994). Moreover, we have shown a strong correlation between ABR threshold shifts and outer hair cell loss (Kaltenbach et al. 1997).

The ANOVAs for ABR threshold shifts indicated significant group differences at all tone pip frequencies: $F(6,41)=4.88,3.35,7.41,23.35$, and 20.02 for the respective frequencies of $2000,4000,8000,16,000$, and $20,000 \mathrm{~Hz}$; all $p$ values $<0.009$. Figure 2 shows that CIS- 


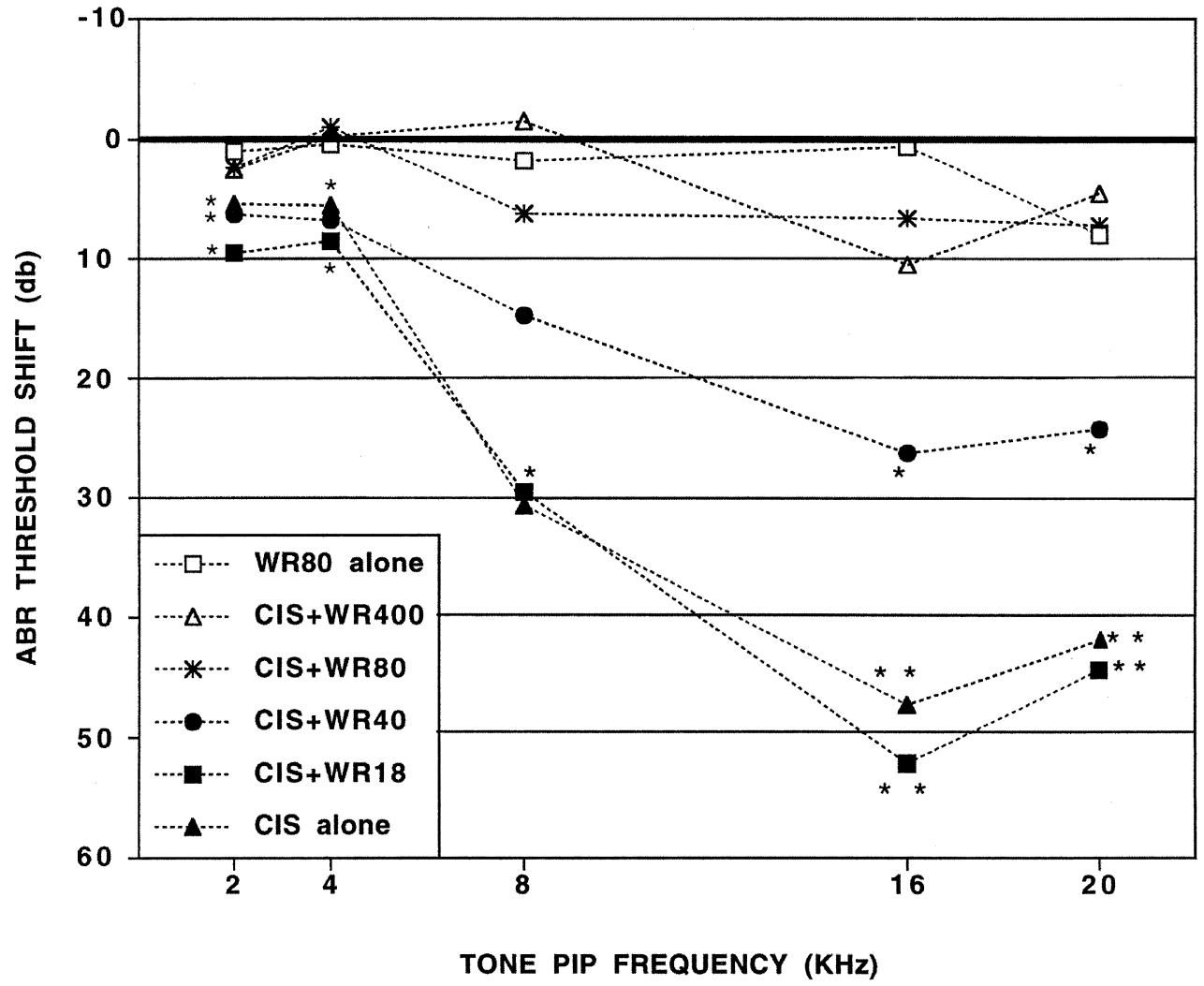

FIG. 2. Mean ABR threshold shifts as functions of treatment condition and tone pip frequency. The Control group's data are represented by the normalized values of $0 \mathrm{~dB}$. The pooled standard error (SE) values for the $2,4,8,16$, and $20 \mathrm{kHz}$ conditions were $0.8,0.9$,

alone caused ABR threshold shifts relative to Control values at all tone pip frequencies and that the degree of ABR threshold shift varied as a function of tone pip frequency. At the lower frequencies of 2000 and $4000 \mathrm{~Hz}$, the CIS-induced ABR threshold shifts were mild: mean $\pm \mathrm{SE}=$

$5.4 \pm 1.8$ and $5.5 \pm 2.3 \mathrm{~dB}$, respectively. The CIS-induced ABR threshold shifts became progressively more dramatic at the higher frequencies of $8000,16,000$, and $2000 \mathrm{~Hz}$ : $\quad$ mean $\pm \mathrm{SE}=30.6 \pm 70, \quad 47.3 \pm 4.9$, and $41.9 \pm 2.6 \mathrm{~dB}$, respectively. Thus, CIS treatment caused a predominantly high-frequency hearing loss. Post hoc pairwise comparisons indicated that the CIS group was significantly different from the Control group at all tone pip frequencies except $4000 \mathrm{~Hz}$.

The mean ABR threshold shifts in the CIS + WR18 group were essentially identical to those observed in the CIS-alone group. This indicated that the WR18 treatment did not ameliorate the CIS-induced hearing loss. Post hoc comparisons indicated that the CIS + WR18 group was significantly different from the Control group at all tone pip frequencies.

The mean ABR threshold shifts in the CIS + WR40 group were less, ranging from about $6 \mathrm{~dB}$ at the lower
2.7, 3.8, and $3.3 \mathrm{~dB}$, respectively; *signifies mean is significantly different from Control values; ${ }^{* *}$ signifies mean is significantly different from corresponding Control and CIS + WR40 values.

frequencies to about $26 \mathrm{~dB}$ at the higher frequencies. This indicated that the WR40 treatment partially ameliorated the CIS-induced hearing loss. Post hoc comparisons indicated that the CIS + WR40 group was significantly different from the Control group at 2000, 16,000, and 20,000 Hz. Because of relatively high variability, these two groups did not differ significantly at $8000 \mathrm{~Hz}$. This high variability was due to some animals exhibiting sizable ABR threshold shifts in response to the $8000 \mathrm{~Hz}$ tone pip, whereas others did not.

The mean ABR threshold shifts in the CIS + WR80 and CIS + WR400 groups were even less dramatic, ranging from none to about $+11 \mathrm{~dB}$. Post hoc comparisons indicated that these two groups did not differ significantly from the Control group at any tone pip frequency. This indicated that the WR80 and WR400 treatments ameliorated the CIS-induced hearing loss almost completely. Thus, WR caused a dose-dependent amelioration of CIS-induced ABR threshold shifts.

There were no significant mean ABR threshold shifts in the WR80-alone group. This indicated that the WR80-alone treatment was not ototoxic. 


\section{ABR latency-intensity profiles}

The plotting of an ABR wave's latency as a function of stimulus intensity is referred to as a latency-intensity $(L-I)$ profile or curve. In normal subjects, latencies gradually prolong as stimulus intensity decreases (Church et al. 1995; Church and Shucard 1986; Jacobson 1994). A subject with a conductive hearing loss will have an elevated ABR threshold and an $L-I$ profile that is displaced to the right and parallel to the normal curve. A subject with a recruitment-type sensorineural hearing loss (SNHL) will also have an elevated ABR threshold. For recruitment-type SNHL, the $L-I$ profile is not parallel to the normal curve, however. Instead, the $L-I$ curve departs progressively from normalcy as stimulus intensity decreases, creating an increasingly steeper than normal slope (Church et al. 1995; Church and Shucard 1986; Jacobson 1994). To develop the $L-I$ profiles, the P1 component was chosen because it reflects activity of the peripheral (auditory) nerve. The latencies of later waves were not used for $L-I$ profile determination because of possible confounding by central pathology.

Figure 3A-C illustrates the effects of the CIS-alone, CIS + WR40, and CIS + WR80 treatments on the $L-I$ profiles in response to the $16,000 \mathrm{~Hz}$ tone pips. Animals in the CIS-alone group showed $L-I$ profiles consistent with moderate to severe recruitment-type SNHL, as depicted in Figure 3A. Specifically, these $L-$ $I$ profiles showed greatly elevated thresholds, prolonged latencies, and extreme steepness in the slopes. The CIS + WR18 data were essentially identical, again reflecting that the WR18 treatment did not ameliorate CIS's ototoxicity. Figures $3 \mathrm{~B}$ and $\mathrm{C}$ illustrate the ameliorative effects of higher WR doses in that animals from the CIS + WR40 and CIS + WR80 groups had $L-I$ profiles that were progressively more normallike in terms of thresholds, latencies, and the steepness of the slopes. The CIS + WR400 data were essentially identical to the CIS + WR80 data. The WR80-alone data were within normal limits.

The $L-I$ profiles for the 8000 and $20,000 \mathrm{~Hz}$ data were highly similar to the $16,000 \mathrm{~Hz}$ shown in Figure 3A-C. At the lower frequencies of 2000 and $4000 \mathrm{~Hz}$, the $L-I$ profiles were normal to near-normal for a large majority of animals receiving CIS-alone or in combination with WR.

\section{ABR interpeak latencies}

In both human and animal studies, the ABR's interpeak latencies (IPLs) are used as measures of brain stem transmission times (BTTs). Prolongations of the IPLs reflect a slowing of the BTT. Prolongations of the IPLs can be caused by brain tumors and demyelinating diseases (Starr and Achor 1975; Stockard and
CIS + WR STUDY

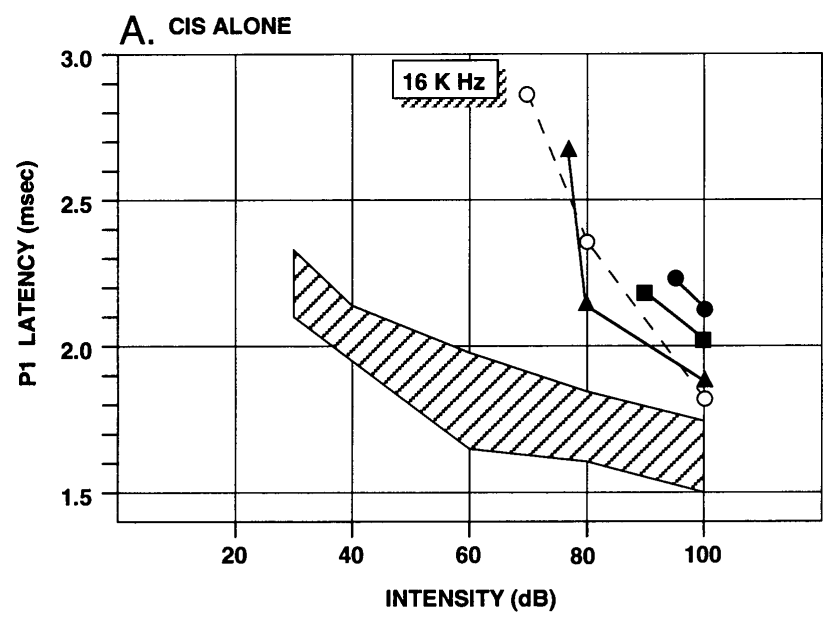

B. $c 15+$ WR 40

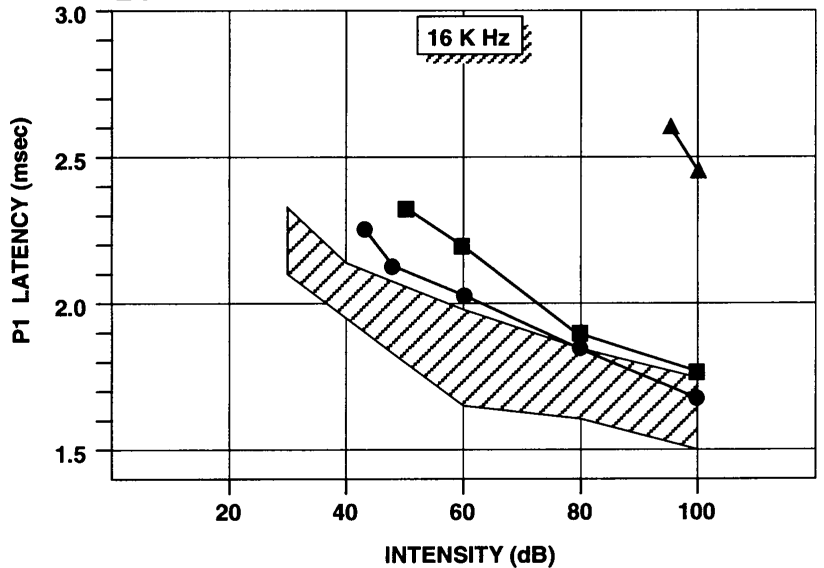

C. $\mathrm{CIS}+$ WR 80

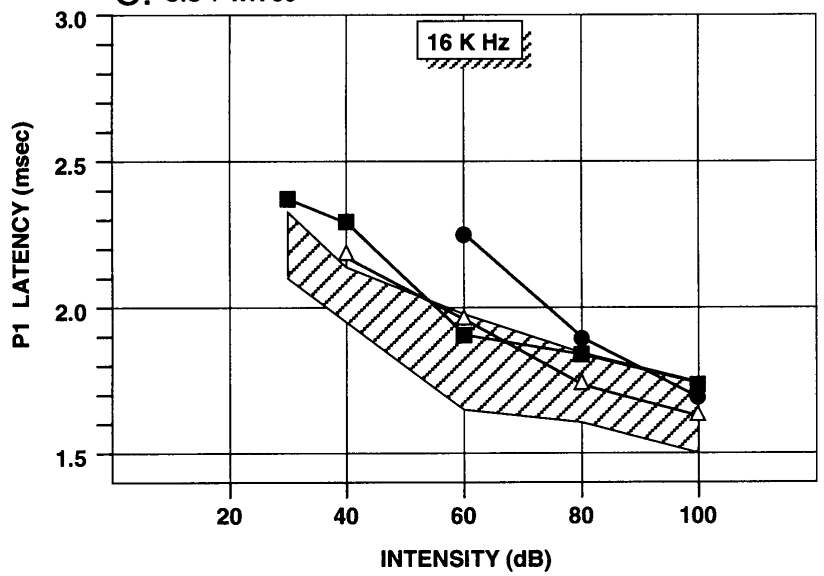

FIG. 3. $A B R$ latency-intensity $(L-l)$ profiles from typical animals in the CIS, CIS + WR40 and CIS + WR80 groups (stimulus $=16,000 \mathrm{~Hz}$ tone pips). The shaded region is the range of normalcy derived from Control data (means $\pm 2 \mathrm{SD}$ ). The thin dark lines show the $L-I$ profiles of individual animals. A. Animals in the CIS group had $L-I$ profiles suggestive of moderate to severe recruitment-type sensorineural hearing loss (SNHL). B, C. Animals in the CIS + WR40 and CIS + WR80 groups showed progressively greater protection from CISinduced SNHL. 


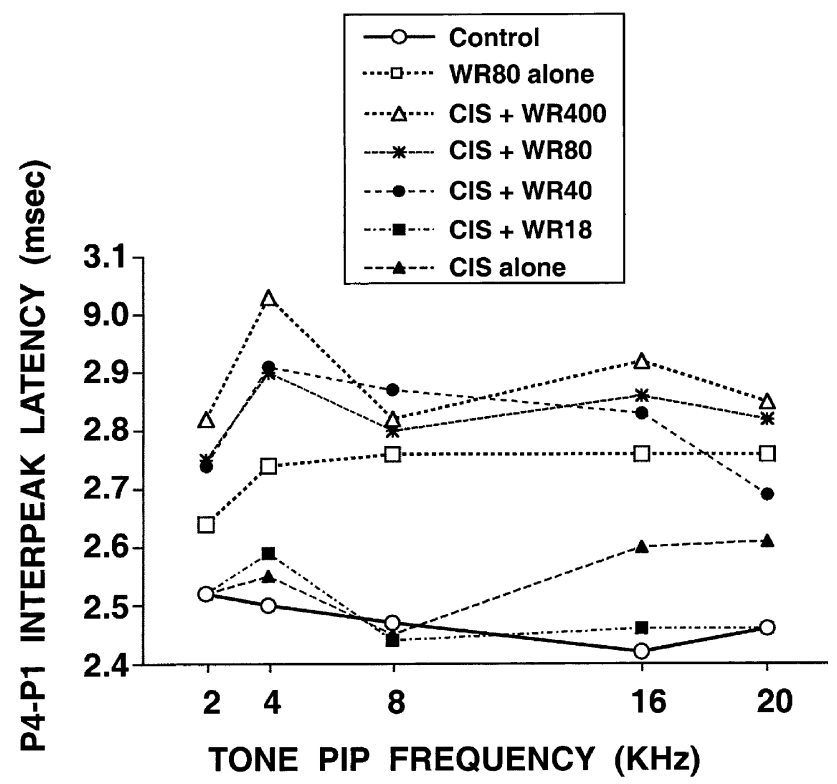

FIG 4. Mean P4-P1 interpeak latencies (IPLs) as functions of treatment condition and tone pip frequency (stimulus intensity $=100$ $\mathrm{dB}$ ). Treatment groups receiving WR doses of $40 \mathrm{mg} / \mathrm{kg}$ or higher had significantly longer P4-P1 IPLs than treatment groups receiving little or no WR treatment. P4-P1 IPL mean values of $2.62 \mathrm{~ms}$ or greater were significantly prolonged relative to the Control group $(p<0.05)$. Standard error (SE) values for the individual means ranged from 0.021 to $0.102 \mathrm{~ms}$.

Rossiter 1977) and neurotoxins (Rebert et al. 1982,1986). Consequently, we examined the hamster's P4-P1 IPL for evidence of both CIS-induced and WR-induced neurotoxicity.

To assess the effects of CIS and WR on the P4-P1 IPL, we analyzed the ABRs elicited by the highest stimulus intensity (i.e., the $100 \mathrm{~dB}$ stimuli) at each tone pip frequency. Only data from the highest stimulus intensity condition were used for the sake of data reduction and because higher stimulus intensities elicit more reliable ABR waveforms. Moreover, $\mathrm{ABR}$ amplitudes and latencies become progressively more normal as stimulus intensity increases in animals and humans with recruitment-type sensorineural hearing losses, as described in the preceding section.

Figure 4 illustrates the hamster P4-P1 IPL as functions of treatment group and tone pip frequency. This figure suggests a dose-dependent effect of WR on the P4-P1 IPL. Specifically, the treatment groups with the higher WR doses (i.e., CIS + WR40, CIS + WR80, CIS + WR400, and WR80-alone) had noticeably longer P4-P1 IPLs than the treatment groups receiving little or no WR (i.e., Control, CISalone, and CIS + WR18). These group differences were verified by a 2-way ANOVA (group $\times$ frequency) which indicated a significant group effect on the P4-P1 IPL: $F(6,198)=61.13 ; p<0.0001$. Post hoc pairwise comparisons of group means indicated that P4-P1 IPLs of $2.62 \mathrm{~ms}$ or greater were significantly prolonged relative to the Control group, regardless of tone pip frequency. This included all the mean IPLs from the CIS + WR40, CIS + WR80, CIS + WR400, and WR80-alone groups. The only exception was the WR80-alone group at the $2000 \mathrm{~Hz}$ condition that was almost significant. Such prolongations of the P4-P1 IPL indicated that the higher WR doses (i.e., the WR40 and higher treatments) had a neurotoxic effect that resulted in the slowing of neural transmission along the brain stem auditory pathway.

The CIS-alone group did not differ significantly from the Control group, indicating no significant neurotoxicity for the CIS-alone condition.

There was a significant main effect for tone pip frequency, indicating that the P4-P1 IPL varied as a function of tone pip frequency: $F(4,198)=4.15 ; p<$ 0.003 . The group-by-frequency interaction was not statistically significant: $F(24,198)=1.38 ; p=0.119$.

\section{DISCUSSION}

This study produced two observations with clinically important implications. Specifically, high doses of WR (Amifostine) were beneficial in protecting against CIS (cisplatin)-induced ototoxicity but harmful in causing neurotoxicity. This is the first study to report either effect.

The present study demonstrated that WR ameliorated CIS-induced ototoxicity in a dose-dependent manner. There was no protection at the low dose of $18 \mathrm{mg} / \mathrm{kg}$, moderate protection at $40 \mathrm{mg} / \mathrm{kg}$, and nearly complete protection at 80 and $400 \mathrm{mg} / \mathrm{kg}$. The finding that WR can protect against CIS-induced hearing loss is contrary to conventional belief. Specifically, several recent human studies reported that WR did not protect patients from CIS-induced hearing loss even though it protected the patients from other forms of toxicity (Gandara et al. 1991; Glover et al. 1987; Kemp et al. 1990; Petrilli et al. 2002; Rick et al. 2001). Our finding of a dosedependent chemoprotective effect for WR on CISinduced hearing loss implies that past human studies used WR doses which were too low to provide protection from CIS's ototoxicity. Although there were no other published accounts of WR providing protection against CIS-induced ototoxicity when we finished our study, we are aware of a recently published ABR study that found such protection when using a WR dose of $1000 \mathrm{mg} / \mathrm{kg} /$ injection in guinea pigs (Hussain et al. 2003). Consequently, higher doses of WR might be considered in future clinical trials. Higher doses of WR might afford better pro- 
tection against CIS's ototoxicity or allow higher doses of CIS to be used.

One potential problem about $\mathrm{WR}$ dose escalation in patients receiving CIS chemotherapy concerns the severity of WR's adverse side effects. WR's adverse side effects in humans include hypotension, nausea and vomiting, hypocalcemia, sneezing, somnolence, dizziness, and chills. These adverse reactions are quite transient, are well tolerated, are easily managed, and rarely require the cessation of treatment. Moreover, there is no evidence that WR interferes with the antitumor efficacy of chemotherapy agents (for review, see Culy and Spencer 2001). Thus, WR dose escalation in future clinical trials would seem to pose little risk and would merit consideration. Militating against this line of thinking is the current study's observation that high doses of WR have the harmful effect of being neurotoxic to the auditory system, at least in the hamster and at the doses used in this study.

Animal studies have investigated the otoprotective effects of several thiol-containing drugs. For example, otoprotection has been observed for sodium thiosulfate (Church et al. 1995; Otto et al. 1988), diethyldithiocarbamate (Church et al. 1995; Rybak et al. 1995), glutathione (Campbell et al. 1996, 2003a), and D-methionine (Campbell et al. 1999, 2003b). To the best of our knowledge, there are only two previously published studies with proper scientific control groups that investigated WR's chemoprotective effects against CIS-induced ototoxicity (Church et al. 1995; Kaltenbach et al. 1997). Both of these studies were by our group, used the relatively low WR dose of $18 \mathrm{mg} / \mathrm{kg}$, and failed to observe rescue from CIS-induced ototoxicity. Our current findings indicate that WR at proper dose levels can protect against CIS-induced ototoxicity in an animal model.

Our study also found that the WR treatments of $40 \mathrm{mg} / \mathrm{kg}$ or higher were neurotoxic in the hamster, as evidenced by prolongations of the ABR's P4-P1 interpeak latencies. The ABR study by Hussain et al. (2003) found a similar effect when using a WR dose of $1000 \mathrm{mg} / \mathrm{kg}$ in guinea pigs. This suggests that the neurotoxic finding is reliable. This also suggests that past failures to observe such an effect were due to a lack of inquiry and/or because the effect occurs only at doses higher than those used in past human and animal studies. Whether other chemoprotective agents have neurotoxic effects on the ABR remains to be investigated.

This neurotoxic effect would argue against using higher doses of WR for achieving otoprotection in humans. However, the results of our study should be further verified in other animal species. Equally important, additional studies need to determine if WR's neurotoxic effects are permanent or temporary. Even though our animals had a relatively long recovery period of 4 weeks, this might not have been an adequate recovery period for WR's neurotoxic effects to fully subside. Future studies should be conducted to study longer recovery periods. In addition, more research is needed to see if WR is causing neurotoxicity in humans at current therapeutic doses, which neural systems are sensitive to this effect, if this effect is permanent or transient, at what dose levels this occurs, and whether or not WR doses can be safely escalated in humans. Because WR can be neurotoxic in hamsters and guinea pigs, it seems imperative to investigate whether the same adverse reaction is occurring in cancer chemotherapy patients receiving WR or other types of chemoprotective treatments.

Several human studies reported that WR acted as a neuroprotectant in that it reduced the neurotoxic side effects of cisplatin (Glover et al. 1989; Kemp et al. 1996; Mollman et al. 1988). At least one study did not see such an effect however (Gelmon et al. 1999). These conflicting findings suggest that more research on WR's neuroprotectant and neurotoxic properties need to be conducted. Perhaps WR acts as a neuroprotectant at relatively moderate doses but is a neurotoxicant at high doses.

The ABR threshold data in Figure 2 indicated that the CIS treatment resulted in mostly a high-frequency hearing loss, with hearing in the lower-frequency ranges being only mildly affected. This result is consistent with past human (e.g., Blakley and Myers 1993; Madasu et al. 1997; Markman et al. 1991; Myers et al. 1991) and animal studies (e.g., Campbell et al. 1999, 2003b; Church et al. 1995; Kaltenbach et al. 1997; Laurell and Bagger-Sjoback 1991; Marco-Algarra et al. 1985; Nakai et al. 1982; Otto et al. 1988; Schweitzer 1993; Schweitzer et al. 1986).

The CIS-alone treatment resulted in ABR $L-I$ (latency-intensity) curves that were consistent with a recruitment-type sensorineural hearing loss SNHL (sensorineural hearing loss). This type of hearing loss is characterized by an $L-I$ function that is steeper than normal, as depicted in Figure 3A. This $L-I$ pattern occurs when the ABR wave latencies are abnormally prolonged at low stimulus intensities but become progressively more normal as stimulus intensity increases (Hood 1998). SNHL occurs when the outer hair cells (OHCs) (sensori-) or the auditory nerve (neural) is the site of the hearing loss. A recruitment-type SNHL refers to an abnormally rapid growth in perceived loudness as sound intensity is raised above an elevated threshold (Ward 1991) or an abnormally large loudness at an elevated threshold (Buus and Florentine 2001). Consistent with this type of hearing loss, histology studies have reported that CIS-induced damage is primarily located in the OHCs, but that such damage can extend to the IHCs 
in severe cases (e.g., Church et al. 1995; Kaltenbach et al. 1997; Laurell and Bagger-Sjoback 1991; MarcoAlgarra et al. 1985; Nakai et al. 1982, Schweitzer 1993). It is interesting to note that the $L-I$ profiles became less steep as the amount of the ABR threshold shift became less. This situation is illustrated in Figures 3B and $\mathrm{C}$ where the higher WR doses ameliorated the CIS-induced effects.

There was a trend for WR to protect against CISinduced mortality. This trend was dose-dependent with no protection at doses of 18 and $40 \mathrm{mg} / \mathrm{kg}$ and complete protection at 80 and $400 \mathrm{mg} / \mathrm{kg}$. This trend $(p=0.098$, 2-sided test $)$ did not quite reach our stochastic criterion level of $p \leq 0.05$. This was probably due to our relatively small group sizes. Power analysis indicated that the study's population size needed to double from $n=54$ to about $n=110$ in order to achieve the criterion level for statistical significance.

In general, doses of $80 \mathrm{mg} / \mathrm{kg}$ or more seemed necessary to provide protection against both ototoxicity and mortality in the hamster. These dosedependent findings are complementary to recent mouse studies that used WR doses of $200-525 \mathrm{mg} / \mathrm{kg}$ to achieve chemoprotection from cisplatin-induced nephrotoxicity, hematotoxicity, gastrointestinal toxicity, and mortality (Ito et al. 1994; Peters et al. 1992; van der Wilt et al. 1992).

CIS treatment in humans can have neurotoxic side effects. This is usually manifested as peripheral neuropathy, especially in the motor and somatosensory nerves (e.g., Thompson et al. 1984). Our study saw no evidence of CIS-induced neurotoxicity in the hamster's ABR. The absence of such an effect implies that the neurogenerators of the hamster's ABR may be resistant to CIS-induced neurotoxicity, that higher CIS doses were needed to produce such an effect, or that the effect was transient and had subsided during the 4-week recovery period.

An ancillary observation was that the WR, by itself and at the doses used in this study, did not cause cochlear hearing loss. Thus, WR does not seem to be ototoxic even at relatively high doses.

In summary, $\mathrm{ABR}$ recordings indicated that high doses of WR were beneficial in protecting against CISinduced ototoxicity but harmful in causing neurotoxicity in hamsters. Escalating WR doses in cancer chemotherapy patients merits consideration because it might ameliorate CIS-induced hearing loss and tinnitus or allow higher doses of CIS to be used. Militating against such a treatment regimen is the possibility that WR dose escalation might cause neurotoxicity in humans. The ABR threshold data and $L-$ I curves clearly indicated that CIS treatment resulted in high-frequency recruitment-type sensorineural hearing losses. Additional findings included: (1) no indication that our CIS treatment caused neurotoxicity to the neurogenerators of the hamster ABR, (2) a trend for higher WR doses to reduce CIS-induced mortality, and (3) WR by itself did not cause a permanent cochlear hearing loss.

\section{ACKNOWLEDGMENTS}

This work was supported by NIH grants R01 DA05536 (MWC), T32-DC00029 (BWB, AG), a Virtual Discovery grant from The Karmanos Cancer Institute (MWC, BWB), and intramural grants from Wayne State University (MWC). We thank Ms. Pamela Holmes and Mr. George Overbeck for their technical assistance.

\section{REFERENCES}

Anthony M, Berg MJ. Biologic and molecular mechanisms for sex differences in pharmacokinetics, pharmacodynamics, and pharmacogenetics: Part II. J. Womens Health Gend. Based Med. 11(7):617-629, 2002.

Blakley BW, Myers SF. Patterns of hearing loss resulting from cisplatinum therapy. Otolaryngol. Head Neck Surg. 109(3 Pt 1):385-391, 1993.

Buus S, Florentine M. Growth of loudness in listeners with cochlear losses: recruitment reconsidered. J. Assoc. Res. Otolaryngol. 3:120-139, 2002.

Campbell KC, Rybak LP, Meech RP, Hughes L. D-Methionine provides excellent protection from cisplatin ototoxicity in the rat. Hear. Res. 102(1-2):90-98, 1996.

Campbell KC, Meech RP, Rybak LP, Hughes LF. D-Methionine protects against cisplatin damage to the stria vascularis. Hear. Res. 138(1-2):13-28, 1999.

Campbell KC, Larsen DL, Meech RP, Rybak LP, Hughes LF. Glutathione ester but not glutathione protects against cisplatin-induced ototoxicity in a rat model. J. Am. Acad. Audiol. 14:124133, 2003a.

Campbell KC, Meech RP, Rybak LP, Hughes LF. The effects of Dmethionine on cochlear oxidative state with and without cisplatin administration: Mechanisms of otoprotection. J. Am. Acad. Audiol. 14:144-156, 2003b.

Church MW, Gritzke R. Effects of ketamine anesthesia on the rat brain-stem auditory evoked potential as a function of dose and stimulus intensity. Electroencephalogr. Clin. Neurophysiol. 67:570-583, 1987.

Church MW, Shucard DW. Age-related hearing loss in BDF1 mice as evidenced by the brainstem auditory evoked potential. Audiology 25:363-372, 1986.

Church MW, Kaltenbach JA, Blakley BW, Burgio DL. The comparative effects of sodium thiosulfate, diethyldithiocarbamate, fosfomycin and WR-2721 on ameliorating cisplatin-induced ototoxicity. Hear. Res. 86:195-203, 1995.

Culy CR, Spencer CM. Amifostine: an update on its clinical status as a cytoprotectant in patients with cancer receiving chemotherapy or radiotherapy and its potential therapeutic application in myelodysplastic syndrome. Drugs 61 (5):641-684, 2001.

Elkind-Hirsch KE, Wallace E, Malinak LR, Jerger JJ. Sex hormones regulate ABR latency. Otolaryngol. Head Neck Surg. 110(1):4652, 1994.

Fuertes MA, Castillab J, Alonsoa C, Perez JM. Cisplatin biochemical mechanism of action: from cytotoxicity to induction of cell death through interconnections between apoptotic and necrotic pathways. Curr. Med. Chem. 10(3):257-266, 2003. 
Gandara DR, Wiebe VJ, Perez EA, Makuch RW, DeGregorio MW. Cisplatin rescue therapy: experience with sodium thiosulfate, WR2721, and diethyldithiocarbamate. Crit. Rev. Oncol. Hematol. 10:353-365, 1990.

Gandara DR, Perez EA, Wiebe V, DeGregorio MW. Cisplatin chemoprotection and rescue; pharmacologic modulation of toxicity. Semin. Oncol. 18(suppl. 3):49-55, 1991.

Gelmon K, Eisenhauer E, Bryce C, Tolcher A, Mayer L, Tomlinson E, Zee B, Blackstein M, Tomiak E, Yau J, Batist G, Fisher B, Iglesias J. Randomized phase II study of high-dose paclitaxel with or without amifostine in patients with metastatic breast cancer. J. Clin. Oncol. 17(10):3038-3047, 1999.

Glover D, Glick J, Weiler C, Fox K, Turrisi A, Kligeman MM. Phase I/II trials of WR-2721 and cis-platinum. Int. J. Radiat. Oncol. Biol. Phys. 12:1509-1512, 1986.

Glover D, Glick JH, Weiler C, Fox K, Guerry D. WR-2721 and highdose cisplatin: an active combination in the treatment of metastatic melanoma. J. Clin. Oncol. 5:574-578, 1987.

Glover D, Gradelsky S, Fox K, Weiler C, Cannon L, Glick J. Clinical trials of WR-2721 and cis-platinum. Int. J. Radiat. Oncol. Biol. Phys. 16(5):1201-1204, 1989.

Hood LJ. Clinical Applications of the Auditory Brainstem Response. Singular Publishing Group, Inc., San Diego, CA, 1998.

Hussain AE, Blakley BW, Nicolas M, Balderston J. Assessment of the protective effects of amifostine against cisplatin-induced toxicity. J. Otolaryngol. 32:294-297, 2003.

Iто H, Kомакі R, Milas L. Protection by WR-2721 against radiation plus cis-diaminedichloroplatinum II caused injury to colonic epithelium in in mice. Int. J. Rad. Oncol. Biol. Phys. 28(4):899903, 1994.

JaCobson JT. Principles and Applications in Auditory Evoked Potentials. Allyn and Bacon, Boston, MA, 1994.

Kaltenbach JA, Church MW, Blakley BW, McCaslin DL, Burgio DL. Comparison of five agents in protecting the cochlea against the ototoxic effect of cisplatin in the hamster. Otolaryngol. Head Neck Surg. 117:493-500, 1997.

Kemp GM, Glover DJ, Schein PS. The role of WR-2721 in the reduction of combined cisplatin and cyclophosphamide toxicity (abstr. 259). Proc. Am. Soc. Clin. Oncol. 9:67.

Kemp G, Rose P, Lurain J, Berman M, Manetta A, Roullet B, Homesley H, Belpomme D, Glick J. Amifostine pretreatment for protection against cyclophosphamide-induced and cisplatin-induced toxicities: results of a randomized control trial in patients with advanced ovarian cancer. J. Clin. Oncol. 14(7):2101-2112, 1996.

Laurell G, Bagger-Sjoback D. Dose-dependent inner ear changes after I.V. administration of cisplatin. J. Otolaryngol. 20:158-167, 1991.

Litterst CL, LeRoy AF, Guarino AM. Disposition and distribution of platinum following parenteral administration of cis-dichlorodiammineplatinum (II) to animals. Cancer Treat. Rep. 63(910):1485-1492, 1979.

LoEhrer PJ, Einhorn LH. Drugs five years later. Cisplatin [review]. Ann. Intern. Med. 100:704-713, 1984.

Madasu R, Ruckenstein MJ, Leake F, Steere E, Robbins KT. Ototoxic effects of supradose cisplatin with sodium thiosulfate neutralization in patients with head and neck cancer. Arch. Otolaryngol. Head Neck Surg. 123(9):978-981, 1997.

Marco-Algarra I, Basterra J, Marco J. Cis-diaminedichloro platinum ototoxicity. Acta Otolaryngol. 99:343-347, 1985.

Markman M, D'Acouisto R, Iannotti N, Kris M, Hakes T, Bajorin D, Bosl G, Reichman B, Casper E, Magill G, , Phase-1 trial of high-dose intravenous cisplatin with simultaneous intravenous sodium thiosulfate. J. Cancer Res. Clin. Oncol. 117(2):151-155, 1991

Moller AR, JEAnNetta PJ. Neural generators of the auditory brainstem response. In: JACOBSON JT (ed) The Auditory Brainstem Response. College-Hill Press, San Diego, CA, pp 13-31, 1985.
Mollman JE, Glover DJ, Hogen WM, Furman RE. Cisplatin neuropathy: risk factors, prognosis, and protection by WR-2721. Cancer 61:2192-2195, 1988.

Myers SF, Blakley BW, Schwan S, Rintelmann WF, Mathog RH. The "plateau effect" of cis-platinum-induced hearing loss. Otolaryngol. Head Neck Surg. 104(1):122-127, 1991.

Nakai Y, Konishi K, Chang KC, Ohashi K, Morisaki N, Minowa Y, Mовiмото A. Ototoxicity of the anticancer drug cisplatin. Acta Otolaryngol 93:227-232, 1982.

Otto WC, Brown RD, Gage-White L, Kupetz S, Anniko M, Penny JE, HeNlEy CM. Effects of cisplatin and thiosulfate upon auditory brainstem responses of guinea pigs. Hear. Res. 35(1):79-85, 1988.

Parham K, Sun XM, KIm DO. Noninvasive assessment of auditory function in mice: auditory brainstem response and distortion product otoacoustic emissions. In: WillotT JP (ed) Handbook of Mouse Auditory Research: From Behavior to Molecular Biology. CRC Press, New York, pp 37-58, 2001.

Peters GJ, van der Wilt CL, Gyergyay F, van Laar JA, Treskes M, VAN der ViJgh WJ, PInedo HM. Protection by WR-2721 of the toxicity induced by the combination of cisplatin and 5-fluorouracil. Int. J. Rad. Oncol. Biol. Phys. 22(4):785-789, 1992.

Petrilli AS, Oliveira DT, Ginani VC, et al. Use of amifostine in the therapy of osteosarcoma in children and adolescents. J. Pediatr. Hematol. Oncol. 24:188-191, 2002.

Pfeifle CE, Howell SB, Felthouse RD, Woliver TB, Andrews PA, Markman M, Murphy MP. High-dose cisplatin with sodium thiosulfate protection. J. Clin. Oncol. 3:237-244, 1985.

Poirier MC, Reed LE, Litterst CL, Katz D, Gupta-Burt S. Persistence of platinum-ammine-DNA adducts in gonads and kidneys of rats and multiple tissues from cancer patients. Cancer Res. 52(1):149-153, 1992.

Rebert CS, Houghton PW, Howd RA, Pryor GT. Effects of hexane on the brainstem auditory response and caudal nerve action potential. Neurobehav. Toxicol. Teratol. 4(1):79-85, 1982.

Rebert CS, Sorenson SS, Pryor GT. Effects of intraperitoneal carbon disulfide on sensory-evoked potentials of Fischer-344 rats. Neurobehav. Toxicol. Teratol. 8(5):543-549, 1986.

Rick O, Beyer J, Schwella N, Schubart H, Schleicher J, Siegert W. Assessment of amifostine as protection from chemotherapy-induced toxicities after conventional-dose and high-dose chemotherapy in patients with germ cell tumor. Ann. Oncol. 12:11511155, 2001.

Rossi GT, BRitT RH. Effects of hypothermia on the cat brain-stem auditory evoked response. Electroencephalogr. Clin. Neurophysiol. 57:143-155, 1984

Rubin JS, Wadler S, Beitler JJ, Haynes H, Rozenblit A, McGill F, Goldberg G, Runowicz C. Audiological findings in a Phase I protocol investigating the effect of WR2721, high-dose cisplatin and radiation therapy in patients with locally advanced cervical carcinoma. J. Laryngol. Otol. 109(8):744-747, 1995.

Russell WM, Burch RL. The Principles of Humane Experimental Techniques. Methuen \& Co., Ltd., London, 1959.

Rybak LP, Ravi R, Somani SM. Mechanism of protection by diethyldithiocarbamate against cisplatin ototoxicity: antioxidant system. Fundam. Appl. Toxicol. 26(2):293-300, 1995.

SchweItzer VG. Cisplatin-induced ototoxicity: The effect of pigmentation and inhibitory agents. Laryngoscope 103:1-52, 1993.

Schweitzer VG, Rarey KE, Dolan DF, Abrams G, Litterst CJ, SheriDAN C. Ototoxicity of cisplatin vs. platinum analogs CBDCA (JM-8) and CHIP (JM-9). Otolaryngol. Head Neck Surg. 94(4):458-470, 1986.

Starr A, Achor J. Auditory brain stem responses in neurological disease. Arch. Neurol. 32(11):761-768, 1975. 
STOCKARD JJ, Rossiter VS. Clinical and pathologic correlates of brain stem auditory response abnormalities. Neurology 27(4):316325, 1977.

Thompson SW, Davis LE, Kornfeld M, Hilgers RD, Standefer JC. Cisplatin neuropathy. Clinical, electrophysiologic, morphologic, and toxicologic studies. Cancer 54(7):1269-1275, 1984.

TRESKES M, VAN DER VIJGH WJ. WR2721 as a modulator of cisplatin-and carboplatin induced side effects in comparison with other chemoprotective agents: a molecular approach. Cancer Chemother. Pharmacol. 33:93-106, 1993.

van der Wilt CL, van Laar JA, Gyergyay F, Smid K, Peters GJ. Biochemical modification of the toxicity and the anti-tumor effect of 5-fluorouracil and cis-platinum by WR-2721 in mice. Eur. J. Cancer 28A(12):2017-2024, 1992.

WARD WD. Noise-induced hearing damage. In: PAPARELLA MM, et al. (eds) Otolaryngology, 3rd ed. WB Saunders Co. Philadelphia, chap 45, p 1643. 